\title{
SPIHT-Based Image Compression Using Optimization of LIS and LIP Encoding
}

\author{
Xianwei Rong, Heting Nie, Weihan Wang, Cunyi Lin, Xiaoyan Yu* \\ School of Physics and Electronic Engineering, Harbin Normal University, 150025, Harbin, China \\ *Corresponding author. Email: yuxiaoyan@hrbnu.edu.cn \\ Manuscript submitted September 10, 2018; accepted November 8, 2018. \\ doi: $10.17706 /$ jcp.13.12.1385-1394
}

\begin{abstract}
This paper presents an optimization scheme for encoding the list of insignificant sets (LIS) and the list of insignificant pixels (LIP) to reduce the redundancies existing in the conventional set partitioning in hierarchical trees (SPIHT) based image compression algorithm. This scheme is based upon the investigation of distribution characteristics of wavelet coefficients. A judgment method with a predetermined threshold value is used for the sorting pass of LIS to identify significant coefficients ahead of encoding the LIS. Moreover, a flag bit representing the number of significant coefficients encoded was introduced for encoding LIP to stop scanning LIP once all the significant coefficients have been encoded. Experimental results with various benchmark images show that the modified SPIHT (MSPIHT) achieves better visual quality and higher Peak Signal to Noise Ratio (PSNR) gains. Furthermore, the number of output 0 bits is significantly decreased at various bit rates compared with the original SPIHT.
\end{abstract}

Keywords: Image compression, wavelet transform, SPIHT, LIP, LIS.

\section{Introduction}

Wavelet transform is widely applied in the image compression due to its excellent time-frequency localization capability and desirable decorrelation ability [1]-[3]. So far, a number of competitive wavelet-based image compression algorithms with an embedded bit stream have been developed [4]-[6], such as Shapiro's embedded zerotree wavelet (EZW), Said and Pearlman's SPIHT, and Taubman's embedded block coding with optimized truncation (EBCOT) [7]-[10]. With an embedded bit stream, the reception of code bits can be stopped at any points and then the image can be reconstructed immediately.

For wavelet-based coding algorithms, EZW and EBCOT require a binary arithmetic coding that increases its hardware cost due to a lot of hardware circuitry and memory. In contrast, SPIHT without arithmetic coding reduces the requirements of memory [11] and possesses much lower computational complexity than EBCOT. Furthermore, it surpasses EZW and is a little bit lower than EBCOT in compression efficiency [12], [13]. Therefore, SPIHT is proverbial for image compression because of its simplicity and efficiency.

Nevertheless, experimental results have indicated that the original SPIHT algorithm endures low coding efficiency [14]-[17] due to that a lot of bits are utilized to encode insignificant coefficients [18]. Moreover, the energy of an original image concentrates on the lowest frequency band of a wavelet-transformed image, whereas all of the wavelet coefficients are encoded comparably by original SPIHT algorithm. Consequently, a large number of redundant 0 bits are generated, which strongly affects its coding efficiency. On the other hand, scanning wavelet coefficients from the largest to the smallest during the process of encoding also consumes many bits for insignificant coefficients. 
In order to address the aforementioned problems, this paper presents an optimization scheme for encoding the LIS and LIP to reduce the redundancy existing in the original SPIHT-based image compression algorithm. According to the investigation of distribution property of wavelet coefficients, a judgment method for testing the significance of LIS is utilized in the sorting pass. Moreover, a flat bit representing the number of significant coefficients encoded is introduced to stop encoding insignificant coefficients in LIP. The experimental results show that the modified SPIHT algorithm achieves improvements by strongly reducing the number of output 0 bits over the original SPIHT for a variety of test images in terms of PSNR values and visual quality.

The remainder of the paper is organized as follows: in Section 2 an overview of original SPIHT algorithm is given. Section 3 experimentally investigates the distribution property of wavelet coefficients on various test images and presents the proposed optimization scheme for the LIS and LIP in detail. Simulation results are provided in Section 4, followed by the conclusion of this paper.

\section{Related Work}

Wavelet transform enables a compact multi-resolution representation of the image [19]. It possesses excellent energy compaction property which is suitable for exploiting redundancy in an image to achieve desired compression performance. SPIHT is used to encode an image in the wavelet transformed domain. A wavelet-transformed image can be organized as a spatial orientation tree (SOT) [Fig. 1(a)], where an arrow represents the relationship between a parent and its offspring; the notations $L L_{n}, H L_{n}, L H_{n}$ and $H H_{n}$ denote the output channels from the $n$th stage; $\mathrm{C}(i, j)$ represents the wavelet coefficient of node $(i, j)$ in the wavelet tree; $\mathrm{O}(i, j)$ means the set of coordinates of all offsprings of node $(i, j) ; \mathrm{D}(i, j)$ and $\mathrm{L}(i, j)$ indicate the set of coordinates of all descendants of node $(i, j)$ and one except for offspring nodes, respectively. The shaded coefficients stand for tree roots. Each node has either four offspring or no offspring. Fig. 1 (b) shows the Morton scanning order of the SOT [20] where the number assigned to each pixel represents the scanning order.
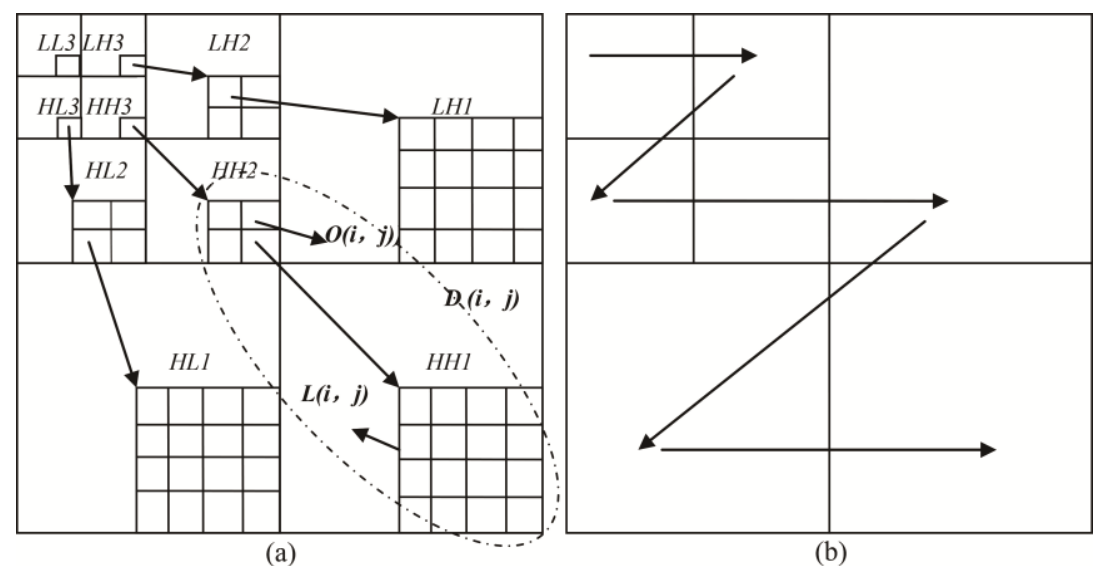

Fig. 1. Principle of SPIHT algorithm. (a) Spatial orientation tree. (b) Morton scanning order of a $16 \times 16$ 3-level wavelet-transformed image.

The significance of set $\tau$ in the $n$th bit-plane is defined as

$$
S_{n}(\tau)=\left\{\begin{array}{l}
1, \quad \max \{|c(i, j)|\} \geq 2^{n} \\
0, \quad \text { otherwise }
\end{array}\right.
$$


When $S_{n}(\tau)$ is "0," $\tau$ is an insignificant set; otherwise, a significant set. The initial threshold $T$ is calculated as $2^{n}$. The value of $n$ can be obtained from

$$
n=\left[\log _{2} \max _{(i, j)}\{C(i, j) \mid\}\right]
$$

The coefficients of a wavelet-transformed image are classified into three lists: the list of insignificant pixels (LIP), the list of significant pixels (LSP) and the list of insignificant sets (LIS). Each of them is used to indicate significance or insignificance of these wavelet coefficients.

The SPIHT is composed of two principal steps, namely sorting pass and refinement pass. At the initialization stage, the coefficients in the highest level are added to LIP, merely those with descendants are added to LIS as type-A entries, and LSP as an empty list. During the sorting pass, the SPIHT algorithm first traverses through the LIP, when a coefficient in LIP becomes significant it is moved to the end of the LSP and its sign is encoded. Sequentially, sets D $(i, j)$ and $L(i, j)$ are encoded following the LIS order, and those that become significant are partitioned into subsets. If $\mathrm{D}(i, j)$ is significant, it is partitioned into $\mathrm{L}(i, j)$ and four single coefficients with $(\mathrm{k}, \mathrm{l}) \in \mathrm{O}(i, j)$.Then the set $\mathrm{L}(i, j)$ is added to the end of LIS as type B entry. If $\mathrm{L}(i, j)$ is significant, it is partitioned into four sets $\mathrm{D}(k, l)$ with $(\mathrm{k}, \mathrm{l}) \in O(\mathrm{i}, \mathrm{j})$ that are added to the end of LIS as type A entries. Each coefficient in LSP except the ones added currently is refined in each refinement pass. The SPIHT algorithm then alternates the above procedure by decreasing current threshold by factor of two until desired bit rate is achieved, i.e., the threshold value in half $T=T / 2$.

Table 1. Distribution Property of Wavelet Coefficients in LIS at Different Bit Planes for Various Images

\begin{tabular}{|c|c|c|c|}
\hline \multirow{3}{*}{ Images } & Bit plane (n) & NSC in LIP & NSC in LIS \\
\hline \multirow{4}{*}{ Lena } & 11 & 503 & 0 \\
\cline { 2 - 4 } & 10 & 902 & 0 \\
\cline { 2 - 4 } & 9 & 1040 & 32 \\
\hline \multirow{4}{*}{ Barbara } & 8 & 1023 & 0 \\
\cline { 2 - 4 } & 11 & 368 & 0 \\
\cline { 2 - 4 } & 10 & 905 & 20 \\
\hline \multirow{5}{*}{ Lion } & 9 & 1029 & 0 \\
\cline { 2 - 4 } & 8 & 1185 & 0 \\
\cline { 2 - 4 } & 11 & 503 & 0 \\
\cline { 2 - 4 } & 10 & 1000 & 25 \\
\hline \multirow{5}{*}{ Peppers } & 9 & 1032 & 0 \\
\cline { 2 - 4 } & 8 & 1117 & 0 \\
\cline { 2 - 4 } & 11 & 399 & 0 \\
\hline
\end{tabular}

Note: NSC denotes the number of significant coefficients.

\section{Proposed Method}

Experimentally, it has been noticed that a substantial number of 0 bits for encoding insignificant coefficients heavily influences the coding efficiency. According to this observation, the proposed optimization of LIS and LIP is utilized for the conventional SPIHT which reduces the number of scanning the LIS and LIP, so that more bits are used to encode significant wavelet coefficients and sets preferentially. 


\subsection{Optimization of LIS Encoding}

The energy of an original image focuses on the lowest frequency band of a transformed image. At the initialization stage, the coefficients in the highest level are added to LIP; only those with descendants are added to LIS. According to the property of wavelet transform, it is known that coefficients in LIP are relatively larger than those in LIS. On the other hand, the initial threshold is calculated as $2^{n}, n$ is the largest integer of logarithm of the maximum of all coefficients. However, the coefficients in LIS are scanned and coded with others in LIP equivalently. That dramatically wastes many 0 bits to encode insignificant sets in LIS in case of large threshold values.

In our experiments, the distribution property of wavelet coefficients in LIS at different bit planes were investigated by using a four-level wavelet decomposition bases upon the bior4.4 filter. The experimental results for diverse benchmark images were listed in Table 1. It can be observed that there is no significant coefficient in LIS when the bit plane is greater than 8(The value is varied with changed wavelet filter and levels). Consequently, a substantial amount of 0 bits are output to encode these insignificant sets in LIS for original SPIHT. According to this observation, an additional judgment is presented and used for whole LIS. As shown in Fig. 2 (shaded parts indicate the optimizations), the proposed optimization scheme first judges the significance of all the coefficients in LIS instead of encoding the sets in LIS straightly for original SPIHT. In other words, the maximum coefficient in LIS is compared with the current threshold. Provided that all the coefficients in LIS are insignificant, then it will be deleted, otherwise LIS is encoded as original SPIHT. The threshold value can be preset as original SPIHT according to formula (2) shown in Section 2.

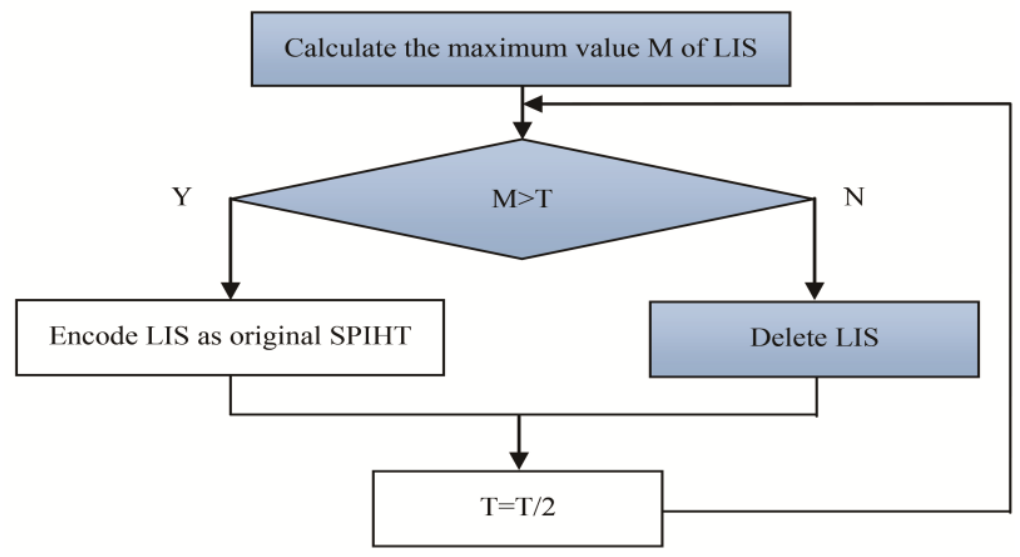

Fig. 2. Flowchart of optimization of LIS encoding.

\subsection{Optimization of LIP Encoding}

For original SPIHT algorithm, wavelet coefficients of $L L_{n} 、 L H_{n} 、 H L_{n}$ and $H H_{n}$ bands are arranged in LIP for $n$ level discrete wavelet transform (DWT). These coefficients in $L L_{n}$ are relatively larger than those in other bands, while all of them in LIP are processed simultaneously. On the other hand, in the view of Morton scanning order of the SOT, the original SPITH first traverses through $L L_{n}$ and then other bands $\left(L H_{n}\right.$ 、 $H L_{n}$ and $H H_{n}$ ). Due to the weakness of LIP, a large quantity of 0 bits unnecessary for accurate reconstruction is encoded in the bitstream.

To exploit the distribution characteristics of wavelet coefficients in LIP at various bit planes, experiments with diverse benchmark images are performed by virtue of a four-level wavelet decomposition based on the bior4.4 filter and the experimental results are listed in Table 2. It is worth noting that there is no significant coefficient in $L L_{n}$ in case of threshold greater than 9 (The value is changed along with the varied 
wavelet filter and levels).

According to the aforementioned investigation, the optimization of LIP is proposed in order to stop encoding insignificant coefficients once all the significant coefficients have been encoded. The main idea of the proposed method is to add a flag bit L representing the number of significant coefficients in LIP encoded, which is shown in Fig. 3 (shaded parts indicate the optimizations). It can be derived from Fig. 3 that the sum of significant coefficients in LIP denoted as S is calculated, and then the coefficients in LIP continue to be encoded until $\mathrm{L}$ is equal to $\mathrm{M}$. The proposed optimization scheme for the original SPIHT is able to save a large number of bits used to encode insignificant coefficients.

Table 2. Distribution of Wavelet Coefficients in LIP at Different Bit Planes for Various Images

\begin{tabular}{|c|c|c|c|}
\hline \multirow{2}{*}{ Images } & Bit plane (n) & NSC in LLn & NSC in LHn、HLn、HHn \\
\hline \multirow{3}{*}{ Lena } & 11 & 503 & 0 \\
\cline { 2 - 4 } & 10 & 912 & 0 \\
\cline { 2 - 4 } & 9 & 1024 & 0 \\
\hline \multirow{3}{*}{ Barbara } & 11 & 369 & 0 \\
\cline { 2 - 4 } & 10 & 905 & 10 \\
\hline \multirow{3}{*}{ Lion } & 9 & 1019 & 0 \\
\cline { 2 - 4 } & 11 & 523 & 0 \\
\cline { 2 - 4 } & 10 & 1000 & 8 \\
\hline \multirow{3}{*}{ Peppers } & 9 & 1032 & 0 \\
\cline { 2 - 4 } & 11 & 399 & 0 \\
\cline { 2 - 4 } & 10 & 755 & 30 \\
\hline
\end{tabular}

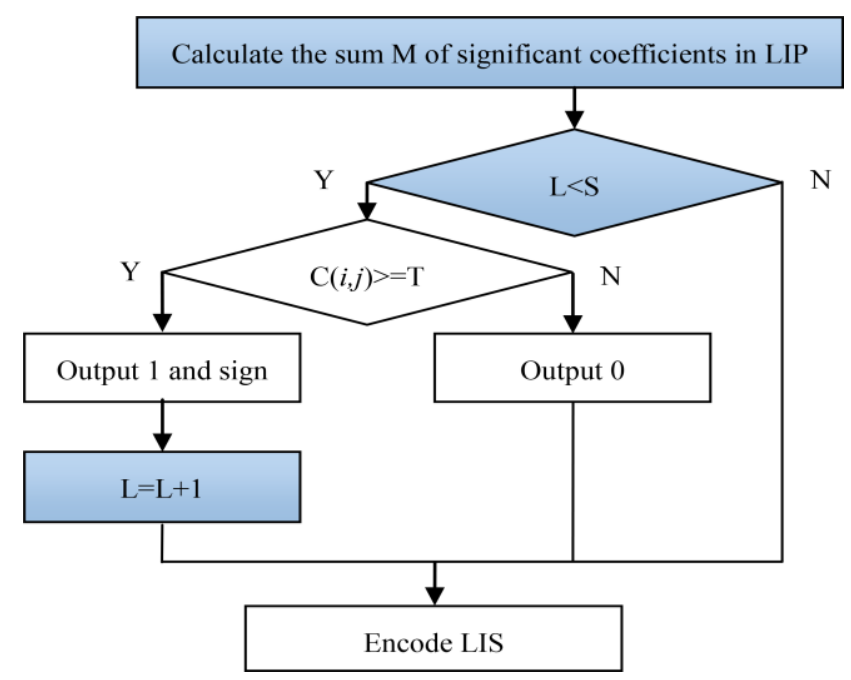

Fig. 3. Flowchart of optimization of LIP encoding.

\section{Simulation Results and Discussion}

In order to evaluate the performance of the modified SPIHT algorithm, simulations were performed using the bior4.4 wavelet filter without entropy coding. Eight 8 -bit grayscale images sized $512 \times 512$, lena, barbara, baboon, peppers, goldhill, couple, boat, crowd, man and milkdrop, are selected as test benchmark. The number of output 0 bits is compared with modified SPIHT and original SPIHT. In addition, a series of 
simulation results are illustrated to measure image quality quantified by PSNR values as well as visual effect of decoded images.

The comparison of output 0 bits are made between modified SPIHT and original SPIHT, which is shown in Table 3. It is evident that modified SPIHT algorithm strongly decreases the number of output 0 bits at different bit rates, so its operation is more efficient than original SPIHT. The image quality can be measured in terms of PSNR values obtained by

$$
P S N R=10 \log _{10}\left(\frac{255^{2}}{\operatorname{MSE}(X)}\right)
$$

where $\operatorname{MSE}(\mathrm{X})$ for image $\mathrm{X}$ denotes the mean-square error, defined as

$$
\operatorname{MSE}(X)=\frac{1}{M N} \sum_{i=0}^{M-1} \sum_{j=0}^{N-1}\left(x[i, j]-x^{\prime}[i, j]\right)^{2}
$$

where $M N$ is the size of an original image, $x(i, j)$ and $x^{\prime}(i, j)$ are the original and reconstructed grayscale values at the pixel $(i, j)$, respectively.

Fig. 4 illustrates several Lena images decoded by modified SPIHT and original SPIHT at different bit rates. It can be derived that modified SPIHT achieves 0.5-5 dB PSNR gains over original SPIHT at low bit rates due to the significant information of original image such as image edge will be encoded preferentially in optimization of LIS and LIP, which clearly demonstrates its enhanced image quality.

Table 3. Performance Comparison between Original SPIHT and Modified SPIHT for Various Images (the

\begin{tabular}{|c|c|c|c|c|c|c|}
\hline Images & $\begin{array}{r}\text { Bit rate } \\
(\mathrm{bpp})\end{array}$ & 0.05 & 0.1 & 0.25 & 0.5 & 1 \\
\hline \multirow{3}{*}{ Lena } & SPIHT & 11259 & 23468 & 52098 & 92742 & 170968 \\
\hline & MSPIHT & 10488 & 21565 & 47699 & 88506 & 166107 \\
\hline & Gain & -771 & -1903 & -4399 & -4236 & -4861 \\
\hline \multirow{3}{*}{ Barbara } & SPIHT & 11083 & 23448 & 50554 & 89942 & 164760 \\
\hline & MSPIHT & 10399 & 21379 & 45495 & 84258 & 160140 \\
\hline & Gain & -684 & -2069 & -5059 & -5657 & -4620 \\
\hline \multirow{3}{*}{ Baboon } & SPIHT & 11273 & 23496 & 51133 & 89114 & 165715 \\
\hline & MSPIHT & 10500 & 21932 & 46124 & 85499 & 160683 \\
\hline & Gain & -773 & -1564 & -5009 & -3615 & -5032 \\
\hline \multirow{3}{*}{ Peppers } & SPIHT & 11573 & 23548 & 52403 & 94527 & 173399 \\
\hline & MSPIHT & 10507 & 21607 & 48644 & 90104 & 169681 \\
\hline & Gain & -1066 & -1941 & -3759 & -4423 & -3718 \\
\hline \multirow{3}{*}{ Goldhill } & SPIHT & 11299 & 23356 & 51172 & 90936 & 167001 \\
\hline & MSPIHT & 10331 & 21513 & 46771 & 85510 & 161778 \\
\hline & Gain & -968 & -1843 & -4401 & -5426 & -5223 \\
\hline \multirow{3}{*}{ Couple } & SPIHT & 11231 & 23438 & 51203 & 91414 & 169505 \\
\hline & MSPIHT & 10411 & 21307 & 47187 & 87057 & 164503 \\
\hline & Gain & -820 & -2131 & -4016 & -4357 & -5002 \\
\hline \multirow{3}{*}{ Boat } & SPIHT & 11303 & 23337 & 51551 & 92177 & 167698 \\
\hline & MSPIHT & 11035 & 22054 & 48494 & 88265 & 164177 \\
\hline & Gain & -268 & -1283 & -3057 & -3912 & -3521 \\
\hline \multirow{3}{*}{ Crowd } & SPIHT & 12811 & 24070 & 53118 & 94070 & 172424 \\
\hline & MSPIHT & 10969 & 21747 & 47635 & 88124 & 166063 \\
\hline & Gain & -1842 & -2323 & -5483 & -5947 & -6361 \\
\hline
\end{tabular}
Output of 0 Bits) 


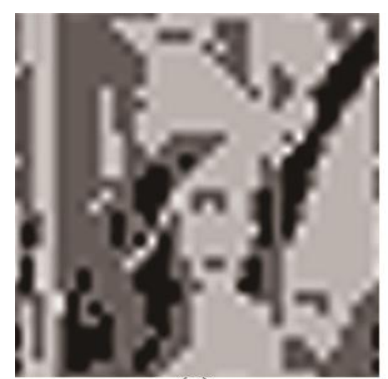

(a)

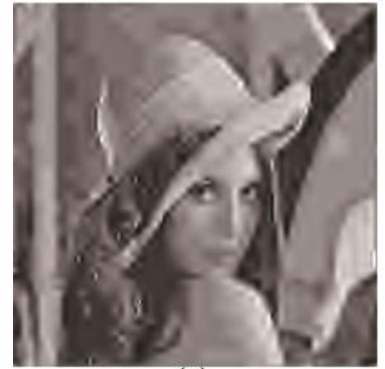

(e)

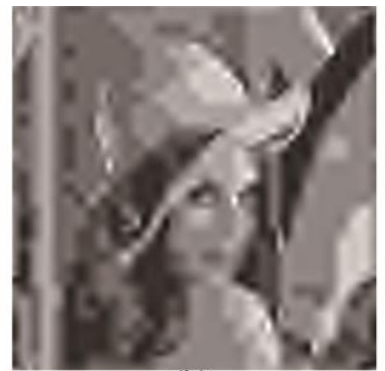

(b)

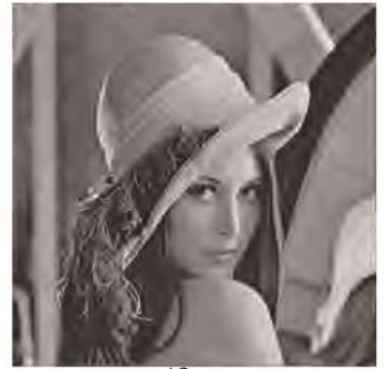

(f)

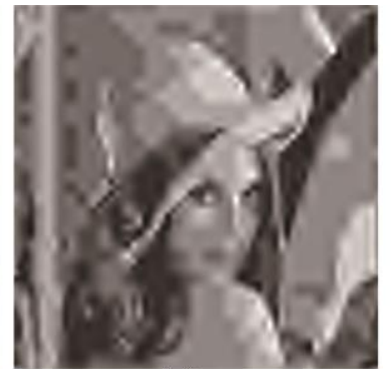

(c)

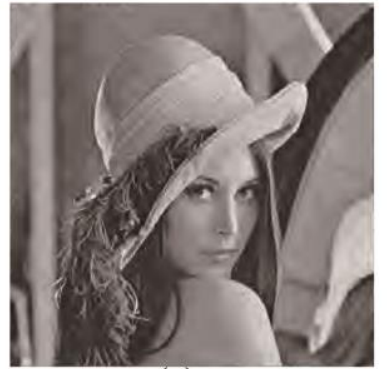

(g)

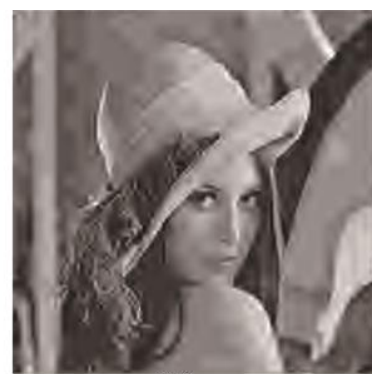

(d)

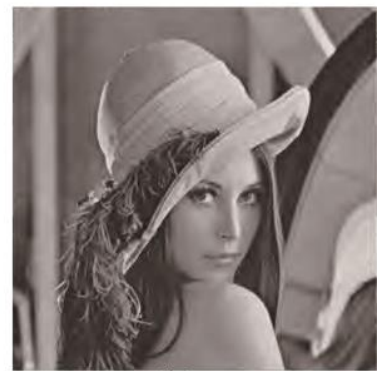

(h)

Fig. 4. Comparison with SPIHT (a-d) and modified SPIHT (e-f) for Lena image. (a) 15.64dB@0.05bpp; (b) 22.29dB@0.15bpp; (c) 24.10dB@0.125bpp;(d)28.29dB@0.2bpp; (e) 21.76dB@0.05bpp; (f) 25.31dB@0.15bpp; (g) 26.94dB@0.125bpp; (h)30.08dB@0.2bpp.
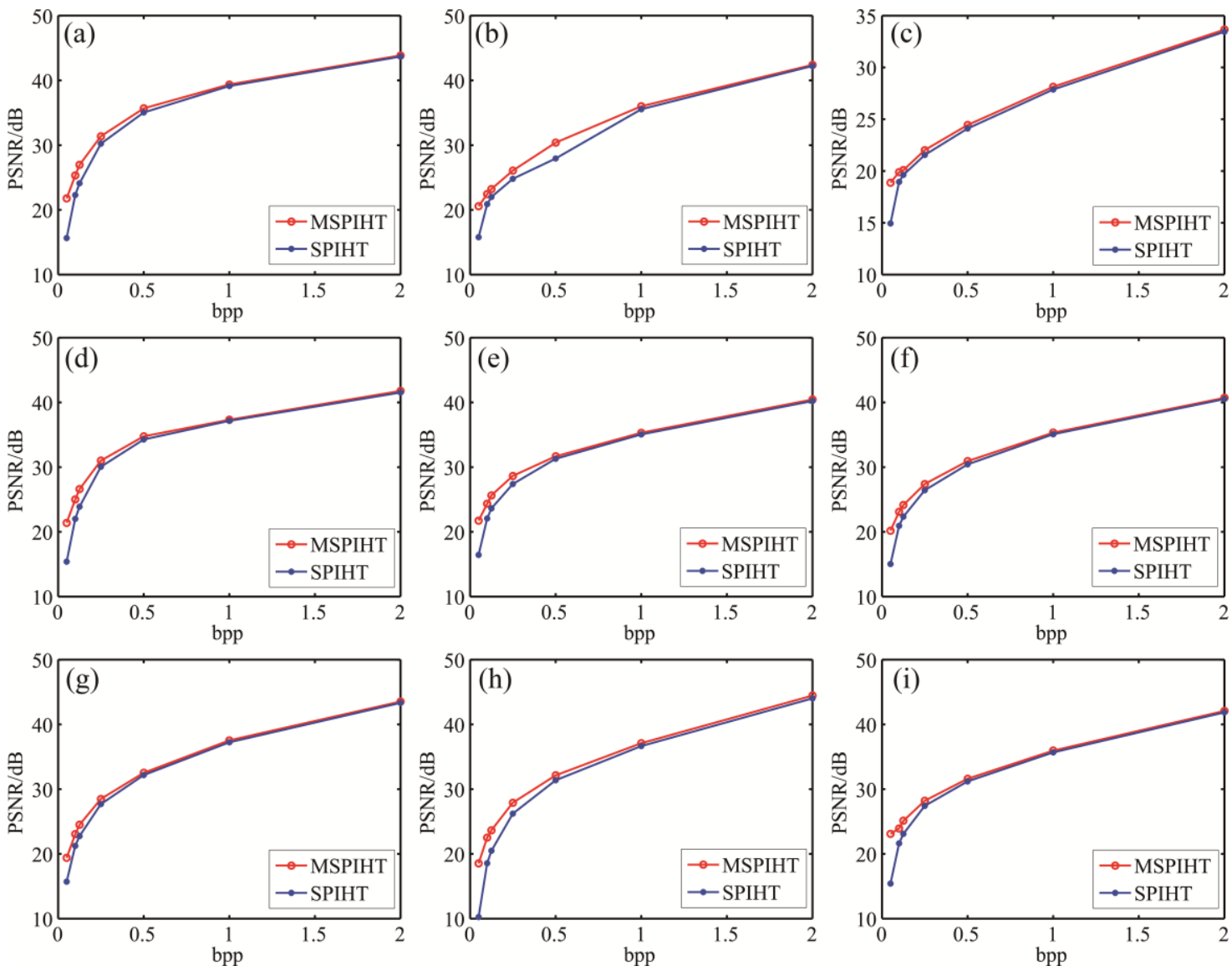

Fig. 5. Comparison with SPIHT and modified SPIHT for various test images. (a) Lena; (b) Barbara; (c) Baboon; (d) Peppers; (e) Goldhill; (f) Couple; (g) Boat; (h) Crowd; (i) Man.

To test the robustness and efficiency of the modified SPIHT, a series of benchmark images with diverse 
characteristics are utilized in the evaluation of image quality. Fig. 5 shows the experimental results in terms of PSNR values produced by original SPIHT and modified SPIHT. It is evident that the modified SPIHT obtains considerable PSNR gains over original SPIHT at a wide range of bit rates for various test images. Moreover, its improvement is not pronounced with increased bit rates due to the fact that modified SPIHT is identical to original SPIHT as the maximum value in LIS is greater than the current threshold.

A number of simulation results mentioned above indicate that modified SPIHT can drastically reduce the number of output 0 bits compared with original SPIHT, so it devotes a much higher percentage of bit streams to significant information than the latter one. Accordingly, it achieves a substantial improvement on image quality at identical bit rates over original SPIHT. Furthermore, images decoded by modified SPIHT can be recognized at very low bit rates.

\section{Conclusion}

In this paper, a new optimization scheme for the LIS and LIP has been proposed to enhance the coding efficiency of the original SPIHT algorithm. The proposed scheme is able to reduce the redundancies by virtue of an additional judgment method, that is, LIS was not encoded until a significant coefficient was covered in it. Moreover, a flag bit was added to the sorting pass of LIP to further reduce the number of output 0 bits. Simulations were performed with a variety of benchmark images and obtained results demonstrated that modified SPIHT algorithm was capable of substantial optimizations on perceptual performance by strongly reducing the number of output 0 bits at various bit rates, especially at low bit rates. Furthermore, the modified SPIHT achieved higher PSNR gains over original SPIHT at distinct bit rates. Nevertheless, the improvement made by the modified SPIHT on PSNR values in the case of high bit rates is not apparent, so we are going to further improve the quality of reconstructed images to satisfy the application requirements.

\section{Acknowledgment}

This work was supported by Educational Agent project of Heilongjiang province (No. 12541225).

\section{References}

[1] Anand, D., Shailendra, S. K., Shabbir, N. M., \& Arun, N C. (2014). High-performance hardware architectures for multi-level lifting-based discrete wavelet transform. EURASIP Journal on Image and Video Processing, 47, 1-19

[2] Isa, S. M., Noviyanto, A., Jatmiko, W., \& Arymurthy, A. M. (2012). The effect of electrocardiogram signal compression using beat reordering and SPIHT on automatic sleep stage classification. Procedia Engineering, 41, 888-896.

[3] Zhang, X., \& Wang, X. (2013). Chaos-based partial encryption of SPIHT coded color images. Signal Processing, 93(9), 2422-2431.

[4] Xiang, T., Qu, J., \& Xiao, (2014). Joint SPIHT compression and selective encryption. Applied Soft Computing, 21, 159-170.

[5] Wang, S., Zheng, D., Zhao, J., Tam, W. J., \& Speranza, F. (2014). Adaptive watermarking and tree structure based image quality estimation. IEEE Trans. Multimedia, 16(2), 311-325.

[6] Lee, K., \& Chung, P. (2013). An attention emphasized bit arrangement in 3-D SPIHT video coding for human vision. J. Vis. Commun. Image, 24(3), 255-269.

[7] Shapiro, J. M. (1993). Embedded image coding using zerotrees of wavelet coefficient. IEEE Trans. Signal Process, 41(12), 2445-2462.

[8] Said, A., \& Pearman, W. A. (1993). A new, fast, and efficient image codec based on set partitioning in hierarchical trees. IEEE Trans. Circuit Syst. Video Technol, 36(6), 243-250. 
[9] Taubman, D. (1993). High Performance scalable image compression with EBCOT. IEEE Trans. Image Process, 44(9), 1158-1170.

[10] Liu, K., Belyaev, E., \& Guo, J. (2012). VLSI architecture of arithmetic coder used in SPIHT. IEEE Trans. VLSI Systems, 20(4), 697-710.

[11] Jin, Y., \& Lee, H. J. (2012). A block-based pass-parallel SPIHT algorithm. IEEE Trans. Circuits Syst. Video Technol, 22(7), 1064-1075.

[12] Said, A., \& Pearlman, W. (1996). A new, fast, and efficient image codec based on set partitioning in hierarchical trees. IEEE Trans. Circuits Syst. Video Technol, 6(3), 243-250.

[13] Pearlman, W. A., Islam, A., Nagaraj, N., \& Said, A. (2004). Efficient, low complexity image coding with a set-partitioning embedded block coder. IEEE Trans. Circuits Syst. Video Technol, 14(11), 1219-1235.

[14] Fang, Z., Xiong, N., Yang, L. T., Sun, X., \& Yang, Y. (2011). Interpolation-based direction-adaptive lifting DWT and modified SPIHT for image compression in multimedia communications. IEEE Systems Journal, 5(4), 584-593.

[15] Saraf, P. D., Sisodia, D., Sinhae, A., \& Sahu, S. (2012). Design and implementation of novel SPIHT algorithm for image compression. Proceedings of the 2nd IEEE Inter. Conf. Parallel Distributed and Grid Computing (PDGC) (pp. 430-434).

[16] Wang, J., \& Cui, Y. (2012). Coefficient statistic based modified SPIHT image compression algorithm. Advances in Computer Science and Information Engineering, 2(169), 595-600.

[17] Chang, S., \& Carin, L. (2006). A modified SPIHT algorithm for image coding with a joint MSE and classification distortion measure. IEEE Trans. Image Process, 15(3), 713-725.

[18] Brahimi, T., Melit, A., \& Khelifi, F. (2009). An improved SPIHT algorithm for lossless image coding. Digital Signal Processing, 19, 220-228.

[19] Antonini, M., Barlaud, M., P. Mathiew, M., \& Daubechies, I. (2004). Image coding using wavelet transform. Proceedings of IEEE Int. Conf. Image Process (pp. 1065-1068).

[20] Algazi, V. R., \& Estes, J. (1995). Analysis-based coding of image transform and subband coefficients. Proceeding of SPIE Vis. Commun. Image Process (pp. 11-21).

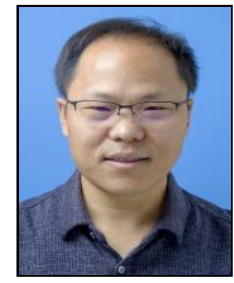

Xianwei Rong was born in Bayan county town, Heilongjiang Province, China, in March 1973. He received his B.S. from the Department of Physics of Harbin Normal University, Harbin, Heilongjiang Province, China, in July 1996. In 2010, he received his M. Eng from School of Information and Communication of Harbin Engineering University.

At present, he is a professor of Harbin Normal University, China. His current research interests focus on image processing and computer vision.

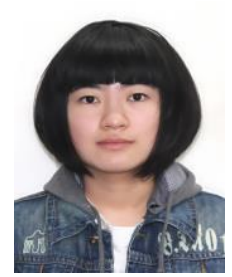

Heting Nie was born in Huhhot city, Nei Mongolia Autonomous Region, China, in March 1990. She received her bachelor degree from the Department of Physics of Baotou Teacher's College, Baotou, Nei Mongolia Autonomous Region China, in July 2012. In 2015, she received her master degree from School of Physics and Electronic Engineering of Harbin Normal University. Her research interests are image processing and computer vision.

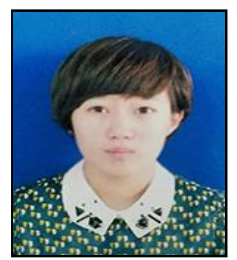

Weihan Wang was born in Jiamusi, Heilongjiang Province, China, in June 1994. She received the B.Eng. degree from the Electronic Information Science and Technology of Harbin Normal University, Harbin, Heilongjiang Province, China, in June 2016.

At present, she is a M.S. candidate at Harbin Normal University, Harbin. Her current research interests are in the areas of image processing and computer vision, in particular image classification and deep learning. 


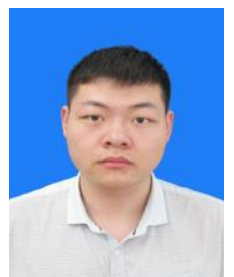

Cunyi Lin was born in Hegang, Heilongjiang Province, China, in February 1995. He received the B.Eng. degree from the Electronic Information Engineering of Anhui University, Hefei, Anhui Province, China, in June 2017.

$\mathrm{He}$ is now a postgraduate student at Harbin Normal University. His current research interests include deep learning and pattern recognition.

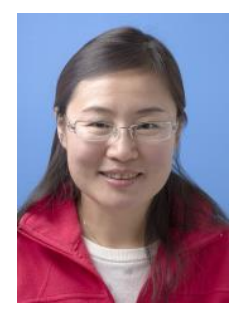

Xiaoyan Yu was born in Huanan county town, Heilongjiang Province, China, in July 1975. She received her B.S. and M.Ed from Harbin Normal University, Harbin, Heilongjiang Province, China, in July 1998 and 2001. In 2006, she received his Ph. D. from Department of Information Systems Engineering of Kochi University of Technology, Kochi, Japan.

At present, she is a professor of Harbin Normal University, China. His current research interests focus on image processing and computer vision. 